(77.5\%) nor of guidelines for dealing with infected athletes $(80.0 \%)$ or for return to sports after a coronavirus infection (88.6\%). Preventive measures mentioned included basic hygiene, measures to reduce personal contacts or virus transmission, or to improve traceability. During confinement, higher age $(p=0.004)$ and training in a club-setting $(p<0.001)$ were associated with reduced sporting activity, while the availability of online training $(p=0.030)$ was linked to increased extent and intensity levels. Lower age $(\mathrm{p}=0.001)$ and recreational sports level $(p=0.005)$ were associated with decreased activity after confinement.

Conclusions While isolation is a necessary measure to protect public health, it also alters physical activity.

\section{THE EFFECTIVENESS OF A VOLLEYBALL INJURY PREVENTION PROGRAM TAILORED TO YOUTH PLAYERS}

${ }^{1}$ Joske Nauta, ${ }^{5}$ Vincent Gouttebarge, ${ }^{3}$ Ellen Kemler, ${ }^{1}$ Matthias Lang, ${ }^{1}$ Ingrid Vriend, ${ }^{4}$ Jacqueline de Wit, ${ }^{1}$ Daniel Zomerdijk, ${ }^{3}$ Victor Zuidema, ${ }^{2}$ Hans Zwerver, ${ }^{1}$ Evert Verhagen. ${ }^{1}$ Department of Public and Occupational Health, Amsterdam UMC, Amsterdam, Netherlands; ${ }^{2}$ Center for Human Movement Sciences, University Medical Center Groningen, University of Groningen, Groningen, Netherlands; ${ }^{3}$ VeiligheidNL - Dutch consumer safety Institute, Amsterdam, Netherlands; ${ }^{4}$ Nevobo - Dutch Volleyball association, Utrecht, Netherlands; ${ }^{5}$ Department of Orthopaedic Surgery, Amsterdam UMC, Amsterdam, Netherlands

\subsection{6/bjsports-2021-IOC.398}

Background In the Netherlands, volleyball is ranked among the top 5 sports with the highest number of injuries, and over $35 \%$ of these injuries are sustained by youth players.

Objective To assess the effectiveness of a volleyball specific youth injury prevention program on injury incidence, injury severity and injury burden.

Design Quasi-experimental study based on a prospective controlled design with a follow-up period of one volleyball season. Randomization was based on competition region (2 intervention regions, 2 control regions). Exposure data of youth volleyball players were collected by trainers on a weekly basis. In case of absence due to a volleyball related injury, injury details were obtained.

Setting Youth teams playing in the Dutch volleyball competition.

Participants Players aged under 17 years who train and compete in the Dutch volleyball. In total, 518 players were included in this study (286 intervention group, 232 control group).

Intervention A volleyball specific warming-up programme tailored to youth, aimed at increasing motor ability and neuromuscular control.

Main Outcome Measurements Injury incidence, injury severity and injury burden. Subgroup analyses for injury onset (acute or overuse) and injury location (upper extremity or lower extremity).

Results No overall effect of the intervention was reported for injury incidence, injury severity and injury burden. Subgroup analysis showed that upper extremity injury rate was reduced by $\sim 60 \%$ (HR $0.41,95 \%$ CI $0.20-0.83$ ). A trend was found suggesting that the risk for an acute injury was reduced by $\sim 40 \%$ (HR 0.61, 95\%CI 0.36 - 1.02).

Conclusions No overall effect of the youth volleyball injury prevention program was found. The preventive program was, however, very effective in reducing upper extremity injury rates in youth players. With respect to acute volleyball related injuries, results were promising.

\section{COMPLIANCE WITH AN INDIVIDUALIZED VS. THE FIFA 11+ INJURY PREVENTION PROGRAM}

${ }^{1}$ Meysam Razi, ${ }^{1}$ Mohammad Hossein Alizadeh, ${ }^{1}$ Hooman Minoonejad, ${ }^{2}$ Tim Meyer. ${ }^{1}$ Department of Health and Sport Medicine, Faculty of Sport Sciences, University of Tehran, Tehran, Iran (Islamic Republic of); ${ }^{2}$ Institute of Sports and Preventive Medicine, Saarland University, Saarbrucken, Germany

\subsection{6/bjsports-2021-IOC.399}

Background Players' compliance with injury prevention programs (IPP) can significantly affect the effectiveness of such programs in injury reduction.

Objective To evaluate players' compliance with an individualized IPP to compared to the established FIFA 11+ IPP.

Design Cross-sectional study

Setting Semi-professional football. The data was obtained from a pilot study comparing the effectiveness of individualized vs. generalized (i. e. one-size-fits-all) IPPs.

Participants 72 semi-professional soccer players from 4 clubs (age: $22.3 \pm 4.7$; weight: $75 \pm 10 \mathrm{~kg}$; height: $177 \pm 9 \mathrm{~cm}$ ) participated in the study and were divided into two groups of 1) Individualized and 2) FIFA 11+.

Interventions The individualized group completed 6 weeks of unsupervised, individualized IPP based on their functional movement screen scores. FIFA $11+$ group completed 6 weeks of FIFA $11+$. After the 6 weeks (18 sessions), the compliance with programs was assessed using an online questionnaire.

Main Outcome Measurements Compliance with the IPP defined as the players' self-assessment of how correctly they followed all instructions during all sessions. Players were classified based on their self-report as fully complied (carried out 16 and more successful sessions), partially complaint (8-15 sessions) and not complaint (less than 8 session).

Results A total of 29 (81\%) players in the individualized PP group said they had fully complied during the 6 weeks of individualized IPP; 5 (14\%) said they had been partially compliant and $2(5 \%)$ classified as not compliant. In FIFA $11+$ group 15 (41\%) had fully complied; 14 (39\%) said they had been partially compliant and $7(20 \%)$ were classified as not compliant.

Conclusions The results indicate that an individualized IPP may be superior to $11+$ IPP in terms of players' compliance. Further research needs to expand the sample size and to investigate the relationship between compliance and injury reduction effect of individualized IPPs.

\section{HOME-BASED INJURY PREVENTION PROGRAM DURING COVID-19 LOCKDOWN REDUCED INJURIES DURING THE REMAINING SEASON IN SEMI-PROFESSIONAL FOOTBALL PLAYERS} ${ }^{1}$ Department of Health and Sport Medicine, Faculty of Sport Sciences, University of Tehran, Tehran, Iran (Islamic Republic of); 'Institute of Sports and Preventive Medicine, Saarland University, Saarbrucken, Germany

10.1136/bjsports-2021-IOC.400

Background The Covid-19 pandemic caused a forced lockdown longer than the normal annual season break in Iranian 